\title{
Study on Distribution Network Reconfiguration with Various DGs
}

\author{
Shengsuo Niu ${ }^{a}$, Yi Ding ${ }^{b}$ and Zhirui Liang ${ }^{c}$ \\ School of Electrical Engineering, North China Electric Power University, Baoding 071003, China \\ aniushengsuo@163.com, bdingyi49@163.com, cliangzr0312@163.com
}

Keywords: distributed generation, network reconfiguration, improved fireworks algorithm, loss.

\begin{abstract}
In order to research the influence of distributed generation access to distribution network, a mathematics model of distribution network reconfiguration with distributed generation is presented in this paper. Fireworks Algorithm is used to solve this problem. According to the characteristic of distributed generations, transforming the reconfiguration to minimum network loss. Let the switching state be the solution vector to achieve the purpose of reduce network loss. IEEE 33 nodes system is selected to make a simulation test, and the results demonstrate that the improved fireworks algorithm can get better convergence effects. Above all, the method in this paper is suitable for optimum the problem of distribution network reconfiguration.
\end{abstract}

\section{Introduction}

In the recent years, the major countries all take their eyes on distributed generation. DG has oceans of differences with traditional centralized power generation, and they mostly are clean energy. So, all of these reason make DG becomes more and more important.

With more and more DG access to the distribution network, the original management and operation will be influenced by them. On this background, the traditional distribution network reconfiguration method is not applicable. Reconfiguration entails altering the topological structure of distribution by changing the status of the switches (sectionalizing switches and tie switches) [1]. We hope that the entire network could reduce power loss, eliminate branches congestion, balance line load and improve voltage quality.

The distribution network reconfiguration problem can be theoretically conclude to a extremely complicated nonlinear integer combination optimization problem [2,3]. Because of switch combination in process of reconfiguration is characteristics of huge quantity, therefore, the single direct solution will fail into the "curse of dimensionality". And due to the solution space of network reconfiguration is tremendous huge. These will cause large amount of calculation in solution process, and couldn't guarantee the reliable convergence. At present, the solving methods of reconfiguration approximately divided into traditional mathematical method, heuristic algorithm and artificial intelligence optimization algorithm.

Although the traditional mathematical method (branch-and-bound method, simplex method, 0-1 integer programming method etc.) is more mature in theory, and can get global optimization without rely on the initial solution of network topology. But this method will easily fail into curse of dimensionality and the consumption of memory is very large. Heuristic algorithm mainly consist of two method, one is optimal flow pattern, and the other is branch exchange method. These two methods improve the calculation speed and reduce the amount of computation, but in some case can't guarantee the global optimum. Artificial intelligence optimization algorithm (particle swarm optimization [4], genetic algorithm [5], bee colony algorithm [6] and bacterial foraging optimization algorithm [7] etc.) has the stronger search capabilities, however, it depends on the initial solution and easily fails into local optimum.

In this paper, improved fireworks optimization algorithm is proposed to solve the problem of distribution network reconfiguration with various DGs. In the iterative process of this algorithm, take the status of switches to be the solution vector. And modified the amplitude equation of fireworks. According to the results of simulation example and existing method, the proposed algorithm offers 
reliable convergence and avoids falling into local optimum, in order to propose a new idea to distribution network reconfiguration with DGs.

Do not number your paper: All manuscripts must be in English, also the table and figure texts, otherwise we cannot publish your paper. Please keep a second copy of your manuscript in your office. When receiving the paper, we assume that the corresponding authors grant us the copyright to use the paper for the book or journal in question.

\section{Problem Formulation}

\subsection{Objective Function of the Problem.}

The objective function of the distribution network reconfiguration always includes decrease distribution network loss, ensure electricity energy quality and balance transmission line load. This paper focus on the economy of system, considering minimum the loss as the objective function.

$$
\min F=\sum_{i=1}^{N} k_{i} R_{i} \frac{P_{i}^{2}+Q_{i}^{2}}{U_{i}^{2}}
$$

where, $F$ is the objective function; $N$ is the number of branch; $R_{i}$ is the resistance of the $i$ th branch; $P_{i}, Q_{i}$ are the active and reactive power inject bus $i ; U_{i}$ is the voltage magnitude at bus $i$; $k_{i}$ is a binary variable which indicates the status of the switch.

\subsection{Constraint Conditions of the Problem.}

The constraint conditions of the distribution network reconfiguration always includes power flow constraint, node voltage constraint, DG capacity constraint, branch capacity constraint and topological structure constraint.

1) Power Flow Constraint

$$
\left\{\begin{array}{l}
P_{i}+P_{D G i}=P_{L i}+U_{i} \sum_{j=1}^{N} U_{j}\left(G_{i j} \cos \delta_{i j}+B_{i j} \sin \theta_{i j}\right) \\
Q_{i}+Q_{D G i}=Q_{L i}+U_{i} \sum_{j=1}^{N} U_{j}\left(G_{i j} \cos \delta_{i j}+B_{i j} \sin \theta_{i j}\right)
\end{array}\right.
$$

2) Node Voltage Constraint

$$
U_{i \min } \leq U_{i} \leq U_{i \max }(i=1,2, \cdots, n)
$$

where, $U_{i \min }$ and $U_{i \max }$ are the voltage limited value at bus $i$.

3) DG Capacity Constraint

$$
\begin{aligned}
& P_{D G \text { imin }} \leq P_{D G i} \leq P_{D G \text { max }} \\
& Q_{D G i \text { min }} \leq Q_{D G i} \leq Q_{D G i \text { max }}
\end{aligned}
$$

where, $P_{D G i}, Q_{D G i}$ are power of the distributed generations at bus $i$.

4) Branch Capacity Constraint

$P_{i}^{2}+Q_{i}^{2} \leq S_{i \max }^{2}$

where, $S_{\text {imax }}$ is the maximum allowable capacity of the branch $i$.

5) Topological Structure Constraint

After the reconfiguration, the topology should be radial status and this paper doesn't consider the isolated island. Literature References

\section{Modeling Various DG}

The traditional network usually contains two node types, $V \theta$ nodes and $P Q$ nodes. But the network with DGs will have some new node types[8, 9]. According to the DG accessing types and inverter types, this paper take various DGs into 4 node types, $P Q$ nodes, $P V$ nodes, $P I$ nodes and $P Q(V)$ nodes.

1) $P Q$ Node Type

$P Q$ node type indicates $P$ and $Q$ are constant. The direct driven synchronous wind turbines are always treat as $P Q$ model. This type of DG can be looked upon as negative load.

2) $P V$ Node Type 
$P V$ node type indicates $P$ and $V$ are constant. The internal combustion and gas turbine which use the synchronous generators are always looked upon as $P V$ model. Because of the power flow calculation method in this paper is forward and backward substitution method, we must take the modification to this type of model:

$$
Q_{i}^{t+1}=Q_{i}^{t}+\Delta Q=Q_{i}^{t}+f\left(\Delta U^{t}\right)=X^{-1} U \Delta U
$$

where, $Q$ is the reactive power of the $P V$ model; $t$ is the iteration number; $f\left(\Delta U^{t}\right)$ is the reactive power modification indicated by voltage deviation.

Finally, the reaction power modification defined as follows:

$$
\begin{cases}Q_{i}^{t+1}=-Q_{i}^{\max } & Q_{i}^{t}+\Delta Q \leq-Q_{i}^{\max } \\ Q_{i}^{t+1}=-Q_{i}^{\min } & Q_{i}^{t}+\Delta Q \geq-Q_{i}^{\min } \\ Q_{i}^{t+1}=Q_{i}^{t}+\Delta Q & -Q_{i}^{\max } \leq Q_{i}^{t}+\Delta Q \leq-Q_{i}^{\min }\end{cases}
$$

3) PI Node Type

$P I$ node type indicates $P$ and $I$ are constant. In general case, the photovoltaic system, fuel cell and micro gas turbine which equipped with current inverter always treat as PI model.

$$
Q_{i}^{t+1}=\sqrt{\left|I_{i}^{2}\right|\left(U^{t}\right)^{2}-P_{i}^{2}}
$$

where, $U^{t}$ is the voltage of the th iteration.

4) $P Q(V)$ Node Type

Fixed speed and slip controlled asynchronous wind turbines which absorb reactive power from power system to build magnetic field don't have the ability of voltage regulation. So, these types of DG are considered as $P Q(V)$ type. The reactive power of $P Q(V)$ model is calculated by $U^{t}$.

$$
\left\{\begin{array}{l}
P_{i}=-P_{s} \\
Q_{i}^{t+1}=-f\left(U^{t}\right)
\end{array}\right.
$$

In this section, this paper take the new node types into PQ model. In this way, the forward and backward substitution method could be used.

\section{Improved Fireworks Algorithm}

\subsection{Overview of Fireworks Algorithm.}

Fireworks algorithm (FWA) is a swarm intelligence based on the fireworks explosion, proposed by Ying Tan and Yuanchun Zhou [10]. The central idea of Fireworks Algorithm is put the optimal problem of solution space treat as a search in the local space around a specific point where the firework is set off through the sparks generated in the explosion. Then calculation the fitness function to get the optimal solution of this iteration. After the numbers of iteration, the final sparks reaches a fairly desired optimum.Fireworks algorithm mainly consist explosion operator, mutation operation, mapping rules and selection strategy.

Stage 1: explosion operator. The number of sparks of fireworks and the amplitude of explosion are defined as follows:

$$
\begin{gathered}
S_{i}=m * \frac{Y_{\max }-f\left(x_{i}\right)+\varepsilon}{\sum_{i=1}^{N}\left(Y_{\max }-f\left(x_{i}\right)\right)+\varepsilon} \\
A_{i}=\hat{A} * \frac{f\left(x_{i}\right)-Y_{\min }+\varepsilon}{\sum_{i=1}^{N}\left(f\left(x_{i}\right)-Y_{\min }\right)+\varepsilon}
\end{gathered}
$$

where, $m$ and $\hat{A}$ is a parameter; $Y_{\max }$ and $Y_{\min }$ are maximum value and minimum value of the fitness function of this swarm; $f\left(x_{i}\right)$ is the fitness value of $x_{i}$; $\varepsilon$ is a smallest constant.

To ensure the number of sparks, we should limit each fireworks:

$$
\hat{s}_{i}= \begin{cases}\operatorname{round}(a \cdot m) & s_{i}<a m \\ \operatorname{round}(b \cdot m) & s_{i}>b m, a<b<1 \\ \operatorname{round}\left(S_{i}\right) & \text { otherwise }\end{cases}
$$


where, round() is integral function; $a$ and $b$ are constant.

Then calculated the displacement of fireworks in the explosion amplitude.

$\Delta x_{i}^{k}=x_{i}^{k}+\operatorname{rand}\left(0, A_{i}\right)$

where, $\operatorname{rand}\left(0, A_{i}\right)$ is random number in explosion amplitude.

Stage 2: mutation operation. To keep the diversity, introducing Gaussian mutation.

$x_{i}^{k}=x_{i}^{k} * N(1,1)$

where, $N(1,1)$ is Gaussian distributions.

Stage 3: mapping rules. If the sparks out of the feasible region, we should put them back through mapping rules.

$x_{i}^{k}=x_{\min }^{k}+\left|x_{i}^{k}\right| \%\left(x_{\max }^{k}-x_{\min }^{k}\right)$

where, $\%$ is modular arithmetic.

Stage 4: selection strategy. After assure the sparks in feasible region, then select the next generation.

$$
R\left(x_{i}\right)=\sum_{j=1}^{K} d\left(x_{i}-x_{j}\right)=\sum_{j=1}^{K}\left\|x_{i}-x_{j}\right\|
$$

where, $R\left(x_{i}\right)$ is the sum of the distances of fireworks which away from $x_{i}$; $K$ is the set of all current locations of both fireworks and sparks. The probabilities of fireworks selection are defined as follows:

$$
p\left(x_{i}\right)=\frac{R\left(x_{i}\right)}{\sum_{j \in K} R\left(x_{j}\right)}
$$

\subsection{Improved Fireworks Algorithm.}

In the original FWA, the fireworks which have better fitness value could explosive more sparks. Correspondingly, the smaller the amplitude of explosion, the better optimization will have. For these reason, this paper modified the amplitude equation of explosion in order to obtain the better optimization speed.

$$
A_{i}=\hat{A}-\frac{t *\left(\hat{A}-A_{\min }\right)}{T}
$$

where, $T$ is constant; $A_{\min }$ is the lower bound of firework explosion amplitude.

The modified amplitude equation can warrant the bigger explosion amplitude in early iteration. However, in the telophase, with the current iteration approaching the maximum number of iterations, algorithm will get the smaller amplitude, that is more focused on the optimization of the best fireworks explode around the point.

\subsection{Improved FWA Applied to Reconfiguration Problem.}

The algorithmic framework to optimize the distribution network reconfiguration problem are presented as follows:

Step 1: Initialization the parameters of distribution network, DGs and the improved FWA algorithm.

Step 2: Randomly generate initial population. Treat the statues of each sectionalizing switches and tie switches as the solution vector.

Step 3: Calculate the power flow through the topology generated by solution vector, and calculate fitness value.

Step 4: Calculate the number of sparks that the firework region according to (12). Through equation (14) and (15) obtain the location of sparks. Select the generation of best fitness value and keep it for the next generation.

Step 5: According to equation (17), randomly select n-1 locations to be the next generation with the selection in step 4 .

Step 6: Calculate the fitness value of new location and update the optimal solution.

Step 7: Check if the iteration meet the maximum value. If meet that value, output the results; if not, go to step 4. 


\section{Results and Discussions}

The proposed method is test on IEEE-33 test system. The bus data for this system are given in [11].To verify the performance of improved FWA and compare with [12] (calculate power flow by [13]) and [14].

Table 1 Reconfiguration results comparison of 3 kinds of algorithm

\begin{tabular}{|c|c|c|c|c|}
\hline \multicolumn{2}{|c|}{ Method } & \multicolumn{2}{|c|}{ Open Switches } & Power Loss $(\mathrm{kW})$ \\
\hline \multicolumn{2}{|c|}{ Initial Network } & \multicolumn{2}{|c|}{$(8,21),(9,15),(12,22),(18,33),(25,29)$} & 202.68 \\
\hline \multicolumn{2}{|c|}{ OFP [12] } & \multicolumn{2}{|c|}{$(7,8),(10,11),(14,15),(32,33),(25,29)$} & 140.28 \\
\hline \multicolumn{2}{|c|}{ Sparsity Leveraging [14] } & \multicolumn{2}{|c|}{$(7,8),(10,11),(14,15),(32,33),(25,29)$} & 140.28 \\
\hline \multicolumn{2}{|c|}{ Improved FWA } & \multicolumn{2}{|c|}{$(7,8),(9,10),(14,15),(32,33),(25,29)$} & 139.56 \\
\hline \multicolumn{5}{|c|}{ Table 2 The related information about DG } \\
\hline \multirow{2}{*}{ Scenario } & \multicolumn{2}{|c|}{ Output Power } & \multirow{2}{*}{$\begin{array}{c}\text { DG candidate bus } \\
\text { number }\end{array}$} & \multirow{2}{*}{ Power Loss $(\mathrm{kW})$} \\
\hline & Active Power & Reactive Power & & \\
\hline \multirow{3}{*}{1} & 0 & 0 & 24 & \multirow{3}{*}{139.5513} \\
\hline & 0 & 0 & 25 & \\
\hline & 0 & 0 & 31 & \\
\hline \multirow{3}{*}{2} & 200 & 0 & 24 & \multirow{3}{*}{97.5918} \\
\hline & 240 & 115 & 25 & \\
\hline & 160 & 75 & 31 & \\
\hline
\end{tabular}

It is observed that the method of [12] and [14] finally optimal the power loss to $140.28 \mathrm{~kW}$, reduced by $30.79 \%$. However, proposed method optimal the power loss reach $139.56 \mathrm{~kW}$, reduced by $31.15 \%$. The minimum voltage is increased from 0.9131p.u. to 0.9378p.u.

To examine the applicability of the proposed method in solving the distribution network reconfiguration with DG. According to some documents, the DG generally access in the relatively heavy load nodes. The DG candidate bus number and output power are shown in table 2.

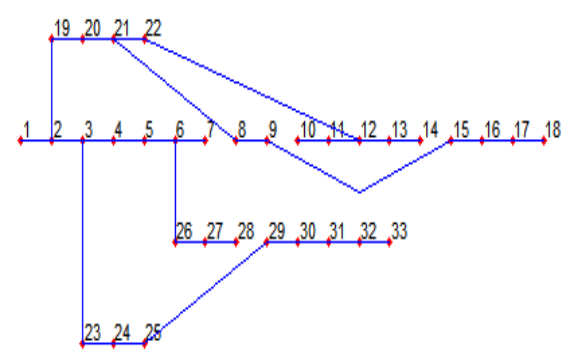

Fig. 1 Reconfiguration topology of scenario 2

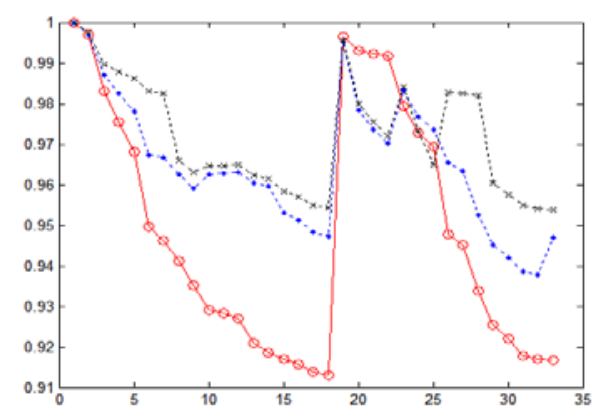

Fig. 2 Comparison of voltage for original network and two scenarios

The reconfiguration results are shown in table 2. And with the same example, the refine adaptive genetic algorithm obtains $100.5316 \mathrm{~kW}$, the particle swarm optimization algorithm obtains 
102.6627kW. According these results, we could know that the proposed method can solve the distribution network reconfiguration problem in a effective way. And from the Fig.2, it shows that after the DGs access to distribution network, they can significantly reduce the system power loss and improve quality of power supply.

\section{Conclusions}

This paper has researched the application of network reconfiguration technique in distribution network with DGs and proposed improved FWA. This algorithm execute multiple point search through simulate fireworks explosion. On this basis, this paper modified the firework amplitude equation, finally realized the target of improving the abilities of global search. The algorithm in this paper is tested in IEEE-33 test system, the results show the applicability and accuracy of proposed algorithm.

\section{References}

[1]. J. Liu, P.X. Bi, W.Y. Yang, et al. Theories of Distribution Systems and Their Applications. China Water \& Power Press, 2007, p. 67-68.

[2]. H.J. Liu, L.C. Li, C.S. Zhang. Reconfiguration of Distribution Network with Distributed Generations Based on Various Load Modes. Power System Protection and Control. Vol.40 (2007) No.11, p. 117-121.

[3]. J.J. Zhao, X. Li, Y. Peng, et al. A Comprehensive Optimization Algorithm for Injection Power of Distributed Generation and Distribution Network Reconfiguration Based on Particle Swarm Optimization. Power System Technology. Vol.33 (2009) No.17, p. 162-166.

[4]. Z.K. Li, X.Y. Chen, K. Yu, et al. Hybrid Particles Swarm Optimization for Distribution Network Reconfiguration. Proceedings of CSEE. Vol.28 (2008) No.31, p. 35-41.

[5]. B.C. Zou, Q.W. Gong, X. Li, et al. Research on Network Reconfiguration GA in Distribution System Based on Load Balancing. Power System Protection and Control. Vol.39 (2011) No.6, p. 80-83.

[6]. M.M. Aman, G.B. Jamson, A.H.A. Bakar, et al. Optimum Network Reconfiguration Based on Maximization of System Loadability Using Continuation Power Flow Theorem. International Journal of Electrical Power \& Energy Systems. Vol.54 (2014), p. 123-133.

[7]. K.S. Kumar, T. Jayabharathi. Power System Reconfiguration and Loss Minimization for an Distribution Systems Using Bacterial Foraging Optimization Algorithm. International Journal of Electrical Power \& Energy Systems. Vol.36 (2012) No.1, p. 13-17.

[8]. W. Wang: Research of Power Flow and Network Loss for the Distribution Network with the Distributed Generation (Master, Lanzhou University, China 2014). p.13.

[9]. M. Ding, X.F. Guo. Three-phase Power Flow for the Weakly Meshed Distribution Network with the Distributed Generation. Advanced in Swarm Intelligence. Vol.29 (2009) No.13, p. 35-40.

[10]. Y. Tan, Y.C. Zhu. Fireworks Algorithm for Optimization. Advances in Swarm Intelligence. Beijing, June 12-15, 2010, p. 355-364.

[11]. Q. Wang: Distribution Network Reconfiguration with Distributed Generation (Master, North China Electric Power University, China 2012). p.33.

[12]. F.V. Gomes, S. Carneiro, J. Pereira, et al. A New Distribution System Reconfiguration Approach Using Optimum Power Flow and Sensitivity Analysis for Loss Reduction. IEEE Transactions on Power Systems. Vol.24 (2006) No.4, p. 2166-2176.

[13]. A.Y. Lam, B. Zhang, A. Dominguez-Garcia, et al. Optimal Distributed Voltage Regulation in Power Distribution Networks. IEEE Transactions on Power Systems. Vol.15 (2012) No.4, p. 1165-1172.

[14]. E. Dall’Anese, G.B. Giannakis. Sparsity Leveraging Reconfiguration of Smart Distribution Systems. IEEE Transactions on Power Delivery. Vol.29 (2014) No.3, p. 1417-1426. 